\title{
Factores asociados a muerte en niños con COVID-19 en México
}

\author{
Rodolfo Rivas-Ruiz, ${ }^{1}$ Ivonne A. Roy-García, ${ }^{1 *}$ Kingston R. Ureña-Wong, ${ }^{2}$ Felipe Aguilar-Ituarte, ${ }^{3}$ \\ Gilberto F. Vázquez-de Anda, ${ }^{4}$ Pedro Gutiérrez-Castrellón, ${ }^{5}$ Javier Mancilla-Ramírez ${ }^{6}$ y \\ Sarbelio Moreno-Espinosa ${ }^{7}$ \\ ${ }^{1}$ Instituto Mexicano del Seguro Social, División de Desarrollo de la Investigación, Centro de Adiestramiento en Investigación Clínica, Ciudad de \\ México; ${ }^{2}$ Asociación para Evitar la Ceguera en México, Coordinación de Enseñanza, Ciudad de México; ${ }^{3}$ Práctica privada, Ciudad de México; \\ ${ }^{4}$ Universidad Autónoma del Estado de México, Facultad de Medicina, Estado de México; ${ }^{5}$ Secretaría de Salud, Hospital "Dr. Manuel Gea González", \\ Centro de Investigación Traslacional en Ciencias de la Salud, Ciudad de México; ${ }^{6}$ Secretaría de Salud, Calidad y Educación en Salud, Ciudad de \\ México; ${ }^{7}$ Secretaría de Salud, Hospital Infantil de México "Federico Gómez", Ciudad de México. México
}

\section{Resumen}

Introducción: Se informa que la mayoría de los niños afectados por SARS-CoV-2 cursan asintomáticos y que en ellos la mortalidad por COVID-19 es baja; en México se desconoce la información al respecto en este grupo de la población. Objetivo: Evaluar los factores de riesgo asociados a mortalidad en niños mexicanos con COVID-19. Método: Análisis secundario de la base de datos de la Dirección General de Epidemiología. Se incluyeron niños menores de 19 años, en quienes se confirmó SARS-CoV-2 mediante RT-PCR. Resultados: Se incluyeron 1443 niños. La mediana de edad fue de ocho años; $3.3 \%$ ingresó a la unidad de cuidados intensivos, $1.8 \%$ requirió ventilación mecánica asistida y la mortalidad fue de $1.9 \%$. En los modelos multivariados, el desarrollo de neumonía constituyó el principal factor de riesgo de mortalidad, con razón de momios (RM) de 6.45 (IC 95 \% 1.99, 20.89); los pacientes que requirieron intubación tuvieron RM de 8.75 (IC 95 \% 3.23, 23.7). Conclusiones: Los niños con COVID 19 tienen alta mortalidad en México, por lo que en ellos se debe procurar evitar la neumonía, especialmente en los menores de cuatro años, con riesgo cardiovascular o inmunosupresión.

PALABRAS CLAVE: Enfermedad por coronavirus 2019. Niños. Mortalidad. Factores de riesgo. Neumonía.

\section{Mortality risk factors in Mexican children with COVID-19}

\begin{abstract}
Introduction: Most children affected by SARS-CoV-2 are reported to be asymptomatic, and COVID-19-related mortality in them is low; in Mexico, there is a lack of information on the subject in this population group. Objective: To assess the risk factors associated with mortality in Mexican children with COVID-19. Method: Secondary analysis of the General Directorate of Epidemiology database. Children younger than 19 years, in whom SARS-CoV-2 infection was confirmed by RT-PCR, were included. Results: 1443 children were included. Median age was eight years; $3.3 \%$ were admitted to the intensive care unit, $1.8 \%$ required assisted mechanical ventilation, and mortality was $1.9 \%$. In multivariate models, the development of pneumonia was the main risk factor for mortality, with an odds ratio (OR) of 6.45 (95\% Cl 1.99, 20.89); patients who required intubation had an OR of 8.75 (95\% Cl 3.23, 23.7). Conclusions: Children with COVID-19 exhibit high mortality in Mexico, and avoiding pneumonia should therefore be tried in them, especially in children younger than four years with cardiovascular risk or immunosuppression.
\end{abstract}

KEY WORDS: Coronavirus disease 2019. Children. Mortality. Risk factors. Pneumonia.

Correspondencia:

*Ivonne A. Roy-García

E-mail: ivonne3316@gmail.com
Gac Med Mex. 2020;156:526-532

Disponible en PubMed

www.gacetamedicademexico.com

0016-3813/๑ 2020 Academia Nacional de Medicina de México, A.C. Publicado por Permanyer. Este es un artículo open access bajo la licencia CC BY-NC-ND (http://creativecommons.org/licenses/by-nc-nd/4.0/). 


\section{Introducción}

En diciembre de 2019, en Wuhan, China, se describió un brote de neumonía, que en poco tiempo se identificó era ocasionado por un nuevo coronavirus, SARS-CoV-2; la enfermedad fue llamada COVID-19. El número de casos de COVID-19 se incrementó y propagó fuera de China y su rápida expansión hizo que la Organización Mundial de la Salud la declarara emergencia sanitaria el 30 de enero de 2020.

El 6 de abril de ese mismo, Estados Unidos reportó el ingreso de 74 niños a unidades de cuidados intensivos pediátricos en 19 estados. En una proyección en el tiempo se estimó que en ese país existirían 176190 niños infectados con SARSCoV-2 (52 381 neonatos y niños menores de dos años, 42857 niños de dos a 11 años y 80952 niños de 12 a 17 años). ${ }^{1}$ Hasta el momento de la elaboración del presente documento existían pocas publicaciones acerca de la afección de la población pediátrica.

Estudios recientes sugieren que los niños son tan propensos como los adultos para infectarse de SARSCoV-2, pero tienen menos síntomas y la enfermedad es menos grave, ${ }^{2}$ como se observa con los coronavirus SARS y MERS-CoV. ${ }^{3,4}$

Existen distintas hipótesis inmunológicas que sustentan los motivos por los cuales los niños tienen menos complicaciones. Una de las más interesantes es la relacionada con el receptor de la enzima convertidora de angiotensina 2 (ECA2), el cual es un sitio de unión del SARS-CoV-2. ${ }^{5}$ Se ha postulado que los niños tienen menos cantidad de receptores ECA2 y, en consecuencia, menor afinidad en comparación con los adultos, por lo cual podrían ser menos afectados. Otra hipótesis es que los niños presentan menos afectación debido a que la mortalidad se ha relacionado con la comorbilidad. ${ }^{6,7}$ Debido a que los niños tienen pocas comorbilidades, se espera que presenten menor morbimortalidad; sin embargo, México es uno de los países con una mayor prevalencia de obesidad infantil, lo cual trae consigo un importante número de pacientes con diabetes, riesgo cardiovascular y enfermedad renal crónica, ${ }^{8}$ por lo que la mortalidad podría ser alta.

Analizar las causas de mortalidad por SARS-CoV-2 en la población pediátrica mexicana y sus factores relacionados, lo cual permitirá mejorar la toma de decisiones en la práctica clínica.

\section{Método}

Se realizó un análisis secundario de la base de datos generada por la Dirección General de Epidemiología, correspondiente a la Secretaría de Salud de México, la cual es una base abierta, actualizada diariamente con los reportes de los casos sospechosos y confirmados mediante prueba positiva para SARS-CoV-2 por el Instituto Nacional de Diagnóstico y Referencia Epidemiológica (https://www. gob.mx/salud/documentos/datos-abiertos-152127).

Se procedió a la descarga de la base, la cual incluyó 215656 pacientes con diagnóstico de COVID-19, actualizada al 23 de mayo de 2020. La base de datos cuenta con las siguientes variables: registro de identificación, origen (en o fuera de Unidades de Salud Monitoras de Enfermedades Respiratorias, no especificado), sector (sector salud o privado), entidad, unidad médica, sexo (hombre o mujer, no especificado), entidad de nacimiento, entidad de residencia, municipio, tipo de paciente (ambulatorio, hospitalizado o no especificado), si es hablante de lengua indígena, nacionalidad (mexicana, extranjera, no especificada), fecha de ingreso, fecha de inicio de síntomas, fecha de defunción (dado el caso), si el paciente se encuentra intubado (en ventilación mecánica), presencia de neumonía, edad, diabetes, asma, inmunosupresiónn, hipertensión arterial generalizada, comorbilidad cardiovascular, obesidad, enfermedad renal crónica, resultado de la prueba para SARS-CoV-2 (positivo, no positivo, pendiente) y si el paciente fue atendido en alguna unidad de cuidados intensivos.

Se seleccionaron los pacientes menores de 18 años con diagnóstico de COVID-19, por la detección del SARS COV-2 mediante prueba de cadena de la polimerasa en tiempo real (RT-PCR).

De las variables mencionadas se procedió a incluir tipo de paciente, fecha de defunción, edad igual o menor de 18 años, diabetes, asma, inmunosupresión, hipertensión arterial generalizada, riesgo cardiovascular alto, obesidad, enfermedad renal crónica, si la atención se llevó a cabo en una unidad de terapia intensiva pediátrica y si se requirió intubación (ventilación mecánica). Estos datos fueron registrados por los médicos tratantes.

\section{Análisis estadístico}

Se limpió la base de datos para buscar datos aberrantes; la edad fue sustituida con un modelo de 
regresión lineal ajustado por sexo en 10 pacientes. Las variables cualitativas (diabetes, asma, obesidad, riesgo cardiovascular y enfermedad renal crónica) mostraron menos de $0.5 \%$ de valores perdidos, los cuales fueron sustituidos por la moda. La variable mortalidad no fue imputada.

Se realizó estadística descriptiva. Para evaluar si la variable edad tenía distribución normal, primero se emplearon pruebas mentales con el objetivo de determinar si la mediana estaba dentro de los intervalos de confianza de la media; si era simétrica y asintótica fue corroborada mediante la prueba de KolmogorovSmirnov, asumiendo un valor de $p>0.05$ como normal. Mediante estas pruebas se definió qué edad tenía libre distribución, por lo que se resumió como mediana y rango intercuartílico (RIC 25-75\%). EI resto de las variables cualitativas se resumieron como frecuencias y porcentajes.

Se llevó a cabo un modelo bivariado en el cual se usó chi cuadrada de Pearson; para evaluar la significación y relevancia clínicas de las variables se calcularon los riesgos relativos (RR) con intervalos de confianza de $95 \%$ (IC $95 \%$ ).

Para los modelos multivariados se usaron las variables que resultaron significativas en el modelo bivariado y se realizó otro modelo clínico en el que se incorporó la variable obesidad, con la finalidad de ajustar por la principal variable de confusión publicada en la literatura mundial. Para el análisis estadístico se utilizaron los softwares $\mathrm{R}$ y SPSS versión 25 . Toda la inferencia estadística fue bilateral (de dos colas).

\section{Resultados}

Se estudiaron 11405 niños con datos clínicos sugerentes de COVID-19, de los cuales 1443 (12.6\%) fueron positivos en la prueba de RT-PCR. Las características generales de ambas poblaciones se resumen en la Tabla 1.

Al analizar las características de los niños con diagnóstico confirmado de SARS-CoV-2 encontramos una mediana de edad de ocho años (RIC 3-14); el sexo predominante fue el femenino. La neumonía se presentó en $9.8 \%$, 3.2 \% tenía antecedente de inmunosupresión, 3.3 ingresó a una unidad de terapia intensiva pediátrica y 1.8 . \% requirió intubación y ventilación mecánica. La mortalidad fue de $1.9 \%$ en comparación con $1.4 \%$ de los niños en quienes no se confirmó el diagnóstico. Los niños positivos a SARS-CoV-2 tuvieron estadísticamente mayor edad, menor frecuencia de neumonía y mayor porcentaje de obesidad (3\% versus $5.7 \%$ ).

En la Tabla 2 se observan los factores de riesgo para mortalidad en los pacientes con prueba de RT-PCR positiva; los principales fueron la edad menor de cuatro años, neumonía, inmunosupresión y riesgo cardiovascular, con RR de 3.8, 53.1, 10.6 y 5.4, respectivamente. El resto de las variables no demostraron resultados estadísticamente significativos, como se aprecia en la Figura 1.

En la Tabla 3 se describe el modelo multivariado, en el que se incluyeron las variables significativas en el modelo bivariado y se consideró a la muerte como variable dependiente. La edad, la inmunosupresión y el riesgo cardiovascular no mostraron ser factores independientes estadísticamente relacionados con la muerte.

El desarrollo de neumonía fue un factor independiente de riesgo, con RM 6.45 (IC $95 \%$ 1.99, 20.89) y la intubación (uso de ventilación mecánica) tuvo una razón de momios de 8.75 (IC 95 \% 3.23, 23.7).

El modelo ajustado conforme la obesidad mostró que esta variable no tuvo efecto significativo en el modelo multivariado, como se aprecia en la Figura 2.

\section{Discusión}

Aquí presentamos valores generales de morbilidad y mortalidad en la población pediátrica mexicana con COVID-19, información que se obtuvo gracias al esfuerzo de los médicos mexicanos y a la Secretaría de Salud de México, que hicieron accesibles los datos para su análisis y publicación.

Durante el primer semestre de la pandemia se registraron 27 niños fallecidos, con una tasa de mortalidad de $1.9 \%$. Esta es la cifra más alta reportada en el mundo, por arriba de la de Estados Unidos, con 26 niños, y el Reino Unido, con 15 casos, y contrasta con la mortalidad de Corea del Sur, donde no se registraron casos..$^{9-14}$ Sin duda, esta alta mortalidad debe alertar a la población, a las asociaciones de pediatría y a la propia Secretaría de Salud.

En este estudio, 11405 niños presentaron diagnóstico sugerente de COVID-19, el cual se confirmó en 1443 niños (12.6\%), de los cuales 1416 presentaron sintomatología leve y fueron tratados ambulatoriamente, $3.3 \%$ ingresó a terapia intensiva y $1.8 \%$ requirió asistencia mecánica para la ventilación. Los niños mexicanos presentaron una evolución más tórpida que en las series informadas en otros países. ${ }^{15,16}$ 
Tabla 1. Características generales de la población pediátrica con diagnóstico de COVID-19

\begin{tabular}{|c|c|c|c|c|}
\hline \multirow[t]{2}{*}{ Variable } & \multicolumn{2}{|c|}{ Sospechosos $(n=11405$} & \multicolumn{2}{|c|}{ Confirmados $(n=1443)$} \\
\hline & Mediana & RIC & Mediana & RIC \\
\hline \multirow[t]{2}{*}{ Edad (años)* } & 8 & $(3-14)$ & 12 & $(5,16)$ \\
\hline & $\mathrm{n}$ & $\%$ & $\mathrm{n}$ & $\%$ \\
\hline Sexo masculino** & 5430 & $(47.6)$ & 693 & $(48)$ \\
\hline Neumonía** & 1683 & $(14.8)$ & 141 & $(9.8)$ \\
\hline Diabetes** & 91 & $(0.8)$ & 16 & $(1.1)$ \\
\hline Asma** & 479 & $(4.2)$ & 49 & (3.4) \\
\hline Inmunosupresión** & 478 & $(4.2)$ & 46 & (3.2) \\
\hline Enfermedad renal crónica** & 95 & $(0.8)$ & 13 & $(0.9)$ \\
\hline Riesgo cardiovascular alto** & 220 & $(1.9)$ & 21 & (1.5) \\
\hline Obesidad $^{\star *}$ & 341 & (3) & 82 & (5.7) \\
\hline Consumo de tabaco** & 118 & (1) & - & - \\
\hline Ingreso a terapia intensiva* & 412 & $(3.6)$ & 48 & (3.3) \\
\hline Intubación** & 294 & $(2.6)$ & 26 & (1.8) \\
\hline Muerte** & 165 & $(1.4)$ & 27 & (1.9) \\
\hline
\end{tabular}

${ }^{*}$ Comparación con $U$ de Mann-Whitney. ${ }^{* *}$ Comparación con $\chi^{2}$.

Los datos resaltados en negritas muestran un valor de $p<0.05$

Tabla 2. Riesgo relativo para predecir mortalidad en pacientes confirmados con COVID-19. Modelo bivariado

\begin{tabular}{|c|c|c|c|c|c|c|c|}
\hline \multirow[t]{2}{*}{ Variable } & \multicolumn{2}{|c|}{ Muertos $(n=27)$} & \multicolumn{2}{|c|}{ Vivos $(n=1416)$} & \multirow[t]{2}{*}{ RR } & \multicolumn{2}{|c|}{ Intervalo de confianza de $95 \%$ (para RR) } \\
\hline & $\mathrm{n}$ & $\%$ & $\mathrm{n}$ & $\%$ & & Límite inferior & Límite superior \\
\hline Edad $<4$ años & 14 & 51.9 & 299 & 21.1 & 3.88 & 1.847 & 8.185 \\
\hline Sexo masculino & 12 & 44.4 & 681 & 48.1 & 0.86 & 0.41 & 1.84 \\
\hline Neumonía & 23 & 85.2 & 118 & 8.3 & 53.1 & 18.62 & 151.3 \\
\hline Diabetes & 0 & 0 & 16 & 1.1 & NC & - & - \\
\hline Asma & 0 & 0 & 49 & 3.5 & NC & - & - \\
\hline Inmunosupresión & 7 & 26 & 39 & 2.8 & 10.6 & 4.7 & 23.9 \\
\hline Enfermedad renal crónica & 0 & 0 & 13 & 1 & $\mathrm{NC}$ & - & - \\
\hline Riesgo cardiovascular alto & 2 & 7.4 & 19 & 1.3 & 5.4 & 1.4 & 21.4 \\
\hline Obesidad & 3 & 11.1 & 79 & 5.6 & 2.1 & 0.64 & 6.75 \\
\hline Ingreso a terapia intensiva & 7 & 26 & 41 & 16.7 & 1.63 & 0.73 & 3.6 \\
\hline Intubación & 12 & 44.4 & 14 & 0.9 & 7.6 & 3.99 & 14.5 \\
\hline
\end{tabular}

A diferencia de lo descrito en influenza, en la que se considera al escolar como el principal diseminador de la enfermedad, ${ }^{17}$ en la pandemia de COVID-19 los niños no desempeñan un papel importante en la transmisión, ya que se ha observado una tasa baja de ataque ( $\mathrm{RM}=0.23$ comparada con la de adultos mayores de 60 años) y en los estudios de familia se ha registrado que solo en $5 \%$ de los casos el caso 
Tabla 3. Análisis multivariado de los principales factores de riesgo asociados a mortalidad en niños con COVID-19

\begin{tabular}{|c|c|c|c|c|c|c|c|}
\hline \multirow[t]{2}{*}{ Variable } & \multicolumn{5}{|c|}{ Niños fallecidos $(n=27)$} & \multicolumn{2}{|c|}{ Intervalo de confianza de $95 \%$ (para RM) } \\
\hline & Mediana & (RIC) & B & EE & RM & Inferior & Superior \\
\hline \multirow[t]{2}{*}{ Edad en años } & 3 & $1-14$ & 0.006 & 0.034 & 1.01 & 0.940 & 1.07 \\
\hline & $\mathrm{n}$ & $\%$ & B & EE & RM & Inferior & Superior \\
\hline Neumonía & 23 & 85.2 & 1.87 & 0.59 & 6.45 & 1.99 & 20.89 \\
\hline Inmunosupresión & 7 & 26 & 0.98 & 0.56 & 2.66 & 0.88 & 8.03 \\
\hline Riesgo cardiovascular alto & 2 & 7.4 & 1.15 & 0.92 & 3.1 & 0.52 & 19.1 \\
\hline Intubación & 12 & 44.4 & 2.17 & 0.63 & 8.75 & 3.23 & 23.7 \\
\hline
\end{tabular}

$\mathrm{EE}=$ error estándar, $\mathrm{RM}=$ razón de momios, $\mathrm{RIC}$ = rango intercuartilar $25-75 \%$.

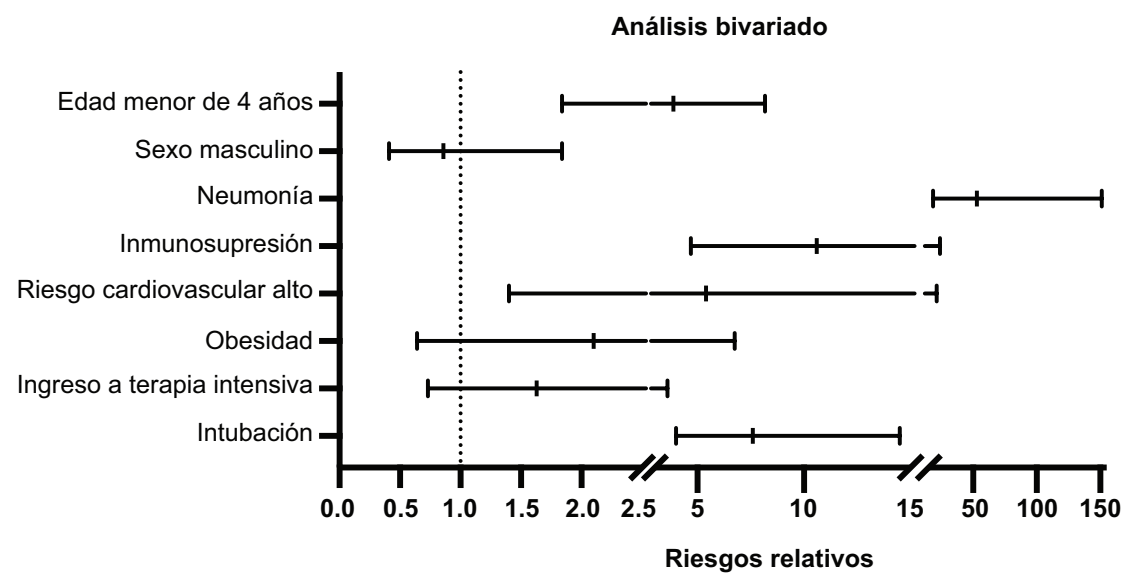

Figura 1. Modelo bivariado de riesgo de muerte en los pacientes con COVID-19 confirmada.

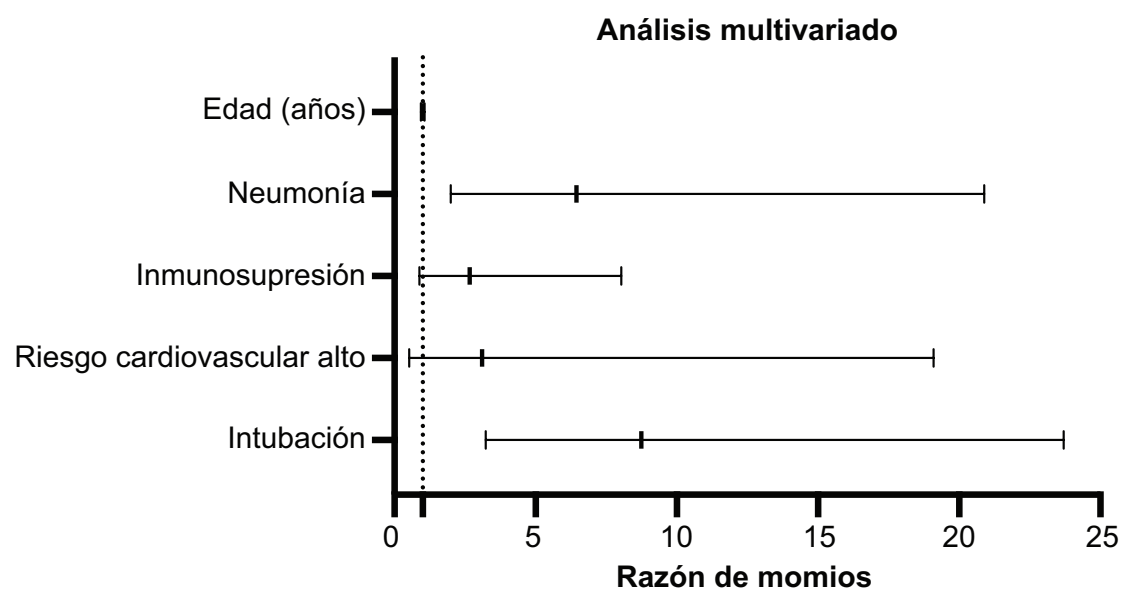

Figura 2. Resultados del modelo multivariado de los niños con COVID-19, en el que la variable dependiente fue la muerte.

índice se trata de un niño. ${ }^{18}$ Además, como la sintomatología en este grupo poblacional es leve, su carga viral es habitualmente baja. ${ }^{19}$ Estos datos son importantes para la planeación de la reincorporación a las actividades escolares.
En una serie de 25 niños en China no se encontró ningún caso de COVID-19 grave. En esa pequeña serie se mencionó que todos los pacientes fueron egresados sin complicaciones. ${ }^{20}$ En nuestro estudio encontramos que la mortalidad se presentó en $1.9 \%$ 
de los niños infectados con SARS-CoV-2, entre quienes los más afectados fueron aquellos con algún tipo de inmunocompromiso o riesgo cardiovascular. Lo anterior corrobora los datos publicados en los que se estima que $3 \%$ ingresará a las terapias intensivas; en nuestro estudio, $43(3.3 \%)$ requirieron ingresar a una unidad de terapia intensiva pediátrica. ${ }^{2}$

Otra serie de nueve casos reportados en China refirió que la neumonía constituyó la manifestación más grave; ninguno de los niños requirió intubación o murió. ${ }^{21}$ Las diferencias entre estas pequeñas series quizá se deben al tamaño de la muestra o a las diferencias en la población infantil entre los países. En México mostramos que la neumonía se presenta en $9.8 \%$ de la población de pacientes con COVID-19. Sin embargo, la neumonía no es mayor que la que presentan los niños sin COVID-19, quienes tienen mayor incidencia de neumonía (14.1\%). No debemos olvidar que el inicio de la pandemia coincidió con el fin de la temporada invernal, en la que son frecuentes las infecciones respiratorias por otros agentes etiológicos como influenza y virus sincicial respiratorio. Esta puede ser la razón de la similitud en las cifras de neumonía por COVID-19 y no COVID-19 en la población pediátrica de México. Se ha reportado que hasta en $30 \%$ de los pacientes sin neumonía clínica se observa imagen radiológica de vidrio despulido, ${ }^{22}$ por lo cual debe definirse la relevancia de la imagen radiológica para establecer el diagnóstico de neumonía.

Sin bien no se presenta mayor frecuencia de neumonía en los pacientes positivos a SARS-CoV-2, sí es más agresiva e incrementa 52 veces más el riesgo de morir que cuando no se desarrolla. Sin duda este dato es crucial, ya que al no haber fármacos eficaces contra SARS-CoV resulta imperativo monitorear estrechamente los requerimientos de oxígeno, buscar la mejor estrategia de apoyo mecánico a la ventilación, identificar marcadores de inflamación, realizar cultivos e identificar otros patógenos respiratorios que pudieran complicar el cuadro de COVID-19. En una cohorte de niños chinos, un estudio multicéntrico europeo y un metaanálisis mundial, el porcentaje de coinfecciones fue de $5.6,5$ y $6 \%$, respectivamente. . $^{9,15,21}$

Aunque no existen ensayos clínicos que validen alguna estrategia farmacológica específica, el costo humano diario de la enfermedad se incrementa día a día, por lo que es preciso el inicio de tratamientos empíricos no completamente demostrados como el uso de antimaláricos, ivermectina, nitazoxanida, azitromicina y retrovirales. ${ }^{23}$
Un aspecto destacable en nuestros resultados es la ausencia de relación entre obesidad y mortalidad, asociación común en adultos pero poco reportada en niños; en una cohorte de niños en Estados Unidos se registró como la principal comorbilidad $(22 \%)^{24}$ y en $15 \%$ de los niños que ingresaron a terapia intensiva por COVID en la Unión Europea y Canadá. ${ }^{11}$ Llama la atención que en este estudio solo se encontró en $5.7 \%$, prevalencia menor a la reportada en la población infantil mexicana, que se estima de $35 \%$. Lo anterior puede atribuirse a que durante la atención en el servicio de urgencias no se registró esta condición.

Una fortaleza de nuestra investigación consistió en el origen de la información. La base de datos de la Dirección General de Epidemiología integra información de todos los pacientes atendidos en las unidades médicas de la República Mexicana, lo que ofrece datos que pueden analizarse para dar pie a investigaciones específicas, con cálculo de tamaño de muestras y mejores diseños, que corroboren nuevas hipótesis.

Una debilidad de esta base de datos es que se cuenta con poca información clínica, la cual incluso pudiera no ser precisa, como el diagnóstico de obesidad o la definición de inmunosupresión o riesgo cardiovascular, lo que favorece sesgos de ejecución. A pesar de ello, permite emitir los primeros pronósticos de la enfermedad y mostrar asociaciones causales con la mortalidad, como el desarrollo de neumonía. Estos datos son necesarios para el mejor entendimiento de COVID-19 y mejorar el tratamiento otorgado a los pacientes pediátricos; ${ }^{25}$ además, podríamos considerarlos un reflejo de la atención actual y de los desenlaces en México.

\section{Conclusiones}

Los niños con COVID-19 tienen menor mortalidad que los adultos; sin embargo, la infección por el SARS-CoV-2 no es benigna y puede tener consecuencias fatales. En los niños con COVID-19 se debe tener especial atención a evitar la neumonía, por lo que el diagnóstico y el tratamiento oportunos, especialmente en niños menores de cuatro años, con riesgo cardiovascular o inmunosuprimidos, podrían ayudar a disminuir la mortalidad.

\section{Conflicto de intereses}

Los autores declaran no tener conflicto de intereses alguno. 


\section{Financiamiento}

Los autores no recibieron patrocinio para llevar a cabo este artículo.

\section{Responsabilidades éticas}

Protección de personas y animales. Los autores declaran que los procedimientos seguidos se apegaron a las normas éticas del comité de experimentación humana responsable y de acuerdo con la Asociación Médica Mundial y la Declaración de Helsinki.

Confidencialidad de los datos. Los autores declaran que siguieron los protocolos de su centro de trabajo en cuanto a la publicación de datos de pacientes.

Derecho a la privacidad y consentimiento informado. En este documento no se indican datos que violen la privacidad ni derechos de los pacientes.

\section{Bibliografía}

1. Pathak EB, Salemi JL, Sobers N, Menard J, Hambleton IR. COVID-19 in children in the United States: intensive care admissions, estimated total infected, and projected numbers of severe pediatric cases in 2020. $J$ Public Health Manag Pract. 2020;26:325-333.

2. Zimmermann P, Curtis N. COVID-19 in children, pregnancy and neonates: a review of epidemiologic and clinical features. Pediatr Infect Dis $\mathrm{J}$ 2020;39:469-477.

3. Leung C, Kwan Y, Ko P, Chiu SS, Loung P, Fong N, et al. Severe acute respiratory syndrome among children. Pediatrics. 2004;113:E535-543.

4. Al-Tawfiq JA, Kattan RF, Memish ZA. Middle East respiratory syndrome coronavirus disease is rare in children: An update from Saudi Arabia. World J Clin Pediatr. 2016:5:391.

5. Letko M, Marzi A, Munster V. Functional assessment of cell entry and receptor usage for SARS-CoV-2 and other lineage B betacoronaviruses. Nat Microbiol. 2020:5:562-569.

6. Patel $A B$, Verma A. COVID-19 and angiotensin-converting enzyme inhibitors and angiotensin receptor blockers: what is the evidence? JAMA 2020:323:1769-1770.

7. Patel AB, Verma A. Nasal ACE2 levels and COVID-19 in children. JAMA. 2020;323:2386-2387.
8. Shamah-Levy T, Cuevas-Nasu L, Gaona-Pineda EB, Gómez-Acosta LM, Morales-Rúan MDC, Hernández-Ávila M, et al. Sobrepeso y obesidad en niños y adolescentes en México, actualización de la Encuesta Nacional de Salud y Nutrición de Medio Camino 2016. Salud Publica Mex. 2018;60:244.

9. Hoang A, Chorath K, Moreira A, Evans M, Burmeister-Morton F, Burmeister $F$, et al. COVID-19 in 7780 pediatric patients: a systematic review. EClinicalMedicine. 2020;24:100433.

10. Castagnoli R, Votto M, Licari A, Brambilla I, Bruno R, Perlini S, et al. Severe acute respiratory syndrome coronavirus 2 (SARS-CoV-2) infection in children and adolescents: a systematic review. JAMA Pediatr. 2020;174:882-889

11. Shekerdemian LS, Mahmood NR, Wolfe KK, Riggs BJ, Ross CE, McKiernan CA, et al. Characteristics and outcomes of children with coronavirus disease 2019 (COVID-19) infection admitted to US and Canadian pediatric intensive care units. JAMA Pediatr. 2020;174:1-6.

12. Korean Society of Infectious Diseases, Korean Society of Pediatric Infectious Diseases, Korean Society of Epidemiology, Korean Society for Antimicrobial Therapy, Korean Society for Healthcare-associated Infection Control and Prevention, Korean Centers for Disease Control and Prevention. Report on the epidemiological features of coronavirus disease 2019 (COVID-19) outbreak in the republic of Korea from January 19 to March 2, 2020. J Korean Med Sci. 2020;35:1-11.

13. Bialek S, Gierke R, Hughes M, McNamara LA, Pilishvili T, Skoff T. Coronavirus disease 2019 in children - United States, February 12-April 2, 2020. MMWR Morb Mortal Wkly Rep. 2020;69:422-426.

14. Khan EA. COVID-19 in children: epidemiology, presentation, diagnosis and management. J Pak Med Assoc. 2020;70:S108-S112.

15. Götzinger F, Santiago-García B, Noguera-Julián A, Lanaspa M, Lancella $\mathrm{L}$, Calò Carducci FI, et al. COVID-19 in children and adolescents in Europe: a multinational, multicentre cohort study. Lancet Child Adolesc Health. 2020;4:1-9.

16. Dong $Y$, Mo X, Hu Y, Qi X, Jiang F, Jiang Z, et al. Epidemiology of COVID-19 among children in China. Pediatrics. 2020;145:e20200702.

17. Neuzil KM, Hohlbein C, Zhu Y. Illness among schoolchildren during influenza season. Arch Pediatr Adolesc Med. 2002;156:986-991.

18. Munro APS, Faust SN. Children are not COVID-19 super spreaders: time to go back to school. Arch Dis Child. 2020;105:618-619.

19. Ludvigsson JF. Children are unlikely to be the main drivers of the COVID-19 pandemic - a systematic review. Acta Paediatr. 2020;109:1-6.

20. Bai K, Liu W, Liu C, Fu Y, Hu J, Qin Y, et al. Clinical analysis of 25 COVID-19 infections in children. Pediatr Infect Dis J. 2020;39:e100-e103.

21. Su L, Ma X, Yu H, Zhang Z, Bian P, Han Y, et al. The different clinical characteristics of corona virus disease cases between children and their families in China-the character of children with COVID-19. Emerg Microbes Infect. 2020;9:707-713.

22. Lu X, Zhang L, Du H, Zhang J, Li YY, Qu J, et al. SARS-CoV-2 infection in children. N Engl J Med. 2020;382:1663-1665.

23. Sanders JM, Monogue ML, Jodlowski TZ, Cutrell JB. Pharmacologic treatments for coronavirus disease 2019 (COVID-19): a review. JAMA. 2020;323:1824-1836.

24. Zachariah $P$, Johnson $C L$, Halabi KC, Ahn D, Sen Al, Fischer A, et al. Epidemiology, clinical features, and disease severity in patients with coronavirus disease 2019 (COVID-19) in a children's hospital in New York City, New York. JAMA Pediatr. 2020;2019:e202430.

25. She J, Liu L, Liu W. COVID-19 epidemic: disease characteristics in children. J Med Virol. 2020;92:747-754. 